Check for updates

1 University of Global Health Equity in Rwanda

2 United Nations University International Institute for Global Health

3 Alliance for Healthy Communities

4 Department of Global Public Health, Karolinska Institutet

5 Consortium of Universities for Global Health

Cite this as: $B M / 2021 ; 375: \mathrm{n} 2658$ http://dx.doi.org/10.1136/bmj.n2658 Published: 01 November 2021

\title{
A call to action to reform academic global health partnerships
}

\author{
Agnes Binagwaho, ${ }^{1}$ Pascale Allotey, ${ }^{2}$ Eugene Sangano, ${ }^{3}$ Anna Mia Ekström, ${ }^{4}$ Keith Martin ${ }^{5}$
}

The global health enterprise has contributed to improving the wellbeing of people and increasing access to health services. However, deep structural inequities persist between institutions from high-income countries (HICs) and those in low and middle-income countries (LMICs) in access to resources, training, and knowledge. This results in significant health inequities, lack of ownership, lost opportunities, misguided priorities, and wholly insufficient attempts at achieving the Sustainable Development Goals.

Power imbalances are embedded across funding opportunities, research management and coordination, knowledge production and transfer, access to training resources and most technical and political aspects of global health. ${ }^{1}$ The current pandemic, which has further highlighted these inequities, is an opportunity to acknowledge and rectify these gaps. ${ }^{2}$ The changes needed include ensuring that partnerships between HIC and LMIC institutions are equitable and that benefits from those arrangements accrue equally to all parties. Collaborations rooted in respect, honesty, equity, as well as commitment to mutual capacity building and health outcomes aligned with the needs of the LMIC partners are essential to reforming global health.

Previous attempts have been made to address this imbalance, but there is a lack of accountability. The following reforms are concrete suggestions, particularly for the academic community, to achieve this objective.

Overcoming the research to policy gap is critical to addressing health challenges. ${ }^{3}$ However, knowledge generated and reported in scientific publications is largely inaccessible to LMIC researchers even when they play a significant role in the research process. ${ }^{4}$ HIC research institutions should provide free access to their academic libraries to their LMIC partners. Moreover, research findings must be shared with equity, fairness, and respect for the work of LMIC and HIC collaborators. Results of global health research should be translated into local languages, with plans drawn at the start to ensure dissemination to all stakeholders including communities which are the subject of the research.

Over $90 \%$ of global health masters programmes (and other associated masters or $\mathrm{PhD}$ programmes) are based in HICs and are utterly unaffordable to students from LMICs. ${ }^{5}$ To bolster the training capacity of LMIC academic institutions, HICs should share their curricula and provide free, online access to $10 \%$ of their students who live and originate from LMICs. This will contribute to LMICs' drive for self-sufficiency.

To facilitate global health education and research, the adoption of technology is critical. Where possible and when needed, HICs should provide free compatible computers, software, and affordable and reliable $3 \mathrm{G}$ broadband to their LMIC partners coupled with training in the use of the technology and access to infrastructure support. ${ }^{6}$

Experiential learning programmes for HIC students are often not equally beneficial to the LMIC host institutions. In line with the goal of mutual benefits, agreements must acknowledge the contributions of LMIC institutions and fully compensate them for the training and use of their assets. HIC institutions could also provide their trainers to build capacity in the host institution as directed by the host. This includes strengthening LMIC management capacity.

Common challenges faced by many LMIC researchers are the lack of grant application skills as well as non-established north-south partnerships among scientists/researchers. ${ }^{7}$ To enable LMIC researchers to collect research funds self-sufficiently, HIC institutions should systematically provide training and mentorship in grant writing and applications as part of all research and teaching partnerships.

Communities and researchers from LMICs are more aware of their most pressing health challenges and the most culturally and locally relevant approaches than their HIC colleagues. ${ }^{8}$ Yet the focus of global health research is often decided by HIC researchers or funding agencies based in HICs. In the future, research questions must align with local needs and must be led by the host institution/LMIC partner. In addition, all research performed in an LMIC must be co-designed in collaboration with local stakeholders. This is critical to challenging the common narrative that knowledge always flows from HICs to LMICs.

Research has shown that LMIC representation in first and last author positions is low, even when all necessary data almost exclusively has been collected and produced by local staff in their own country. ${ }^{9}$ Whenever needed, HIC institutions should provide senior support and training in article writing as well as ensure fair and equitable authorship of research papers between participating scientists from LMICs and HICs.

The covid-19 pandemic has emphasised the importance of partnerships in addressing global health challenges-but also that we have a very long road ahead to make these equitable, mutually beneficial, and respectful of all parties. This call for change is no longer a mere suggestion.

It is a dire request to the global health community to challenge the ingrained and detrimental status quo that is a heritage of the colonial mindset.

If the reforms in this paper are implemented, they would greatly improve our chances of reaching the Sustainable Development Goals and making health 


\section{OPINION}

\section{for all a more realistic and much more sustainable goal.}

Competing interests: none declared

Provenance and peer review: not commissioned, not peer reviewed

1 Olusanya JO, Ubogu Ol, Njokanma FO, Olusanya BO. Transforming global health through equity-driven funding. Nat Med 2021:27:1136-8.

doi: 10.1038/s41591-021-01422-6. pmid: 34168335

2 Jensen N, Kelly AH, Avendano M. The COVID-19 pandemic underscores the need for an equity-focused global health agenda. Humanit Soc Sci Commun 2021;8:15. doi: 10.1057/s41599-020-00700-x

3 Martin K, Mullan Z, Horton R. Overcoming the research to policy gap. Lancet Glob Health 2019;7(Suppl 1):S1-2. doi: 10.1016/S2214-109X(19)30082-8. pmid: 30857615

4 Smith E, Haustein S, Mongeon P, Shu F, Ridde V, Larivière V. Knowledge sharing in global health research - the impact, uptake and cost of open access to scholarly literature. Health Res Policy Syst. 2017;15(1):73. Published 2017 Aug 29. doi: 10.1186/s12961-017-0235-3

5 Svadzian A, Vasquez NA, Abimbola S, Pai M. Global health degrees: at what cost?BMJ Glob Health 2020;5:e003310. doi: 10.1136/bmigh-2020-003310 pmid: 32759185

6 Is technology key to improving global health and education, or just an expensive distraction? https://www.weforum.org/agenda/2019/05/technology-health-education-developing-countries/

7 Morley, Katharine \& Morley, Michael \& Beratarrechea, Andrea. (2020). Funding Global Health Projects. doi: 10.1007/978-3-030-47994-7_5.

8 Zaman M, Afridi G, Ohly H, etal. Equitable partnerships in global health research. Nat Food 2020;1:760-1. doi: 10.1038/543016-020-00201-9.

9 Cash-Gibson L, Rojas-Gualdrón DF, Pericàs JM, Benach J. Inequalities in global health inequalities research: A 50-year bibliometric analysis (1966-2015). PLoS One 2018;13:e0191901.

doi: 10.1371/journal.pone.0191901. pmid: 29385197 